\title{
Outcome of Men Presenting with Clinical Breast Problems: The Role of Mammography and Ultrasound
}

\author{
Stephanie K. Patterson, MD, ${ }^{*}$ Mark A. Helvie, MD, ${ }^{*}$ Khadija Aziz, MD ${ }^{\dagger}$ and Alexis \\ V. Nees, MD* \\ "Department of Radiology, University of Michigan Hospitals, Ann Arbor, Michigan; and ${ }^{\dagger}$ Department of \\ Diagnostic Radiology, University of Kentucky Medical Center, Lexington, Kentucky
}

Abstract: The purpose of this study was to determine the outcome of men presenting with clinical breast problems for breast imaging and to evaluate the role of mammography and ultrasound in the diagnosis of benign and malignant breast problems. We retrospectively reviewed clinical, radiographic, and pathologic records of 165 consecutive symptomatic men presenting to Breast Imaging over a 4 year period. We assessed the clinical indication for referral, mammographic findings, sonographic findings, histologic results, and clinical outcomes. Patients ranged in age from 22 to 96 years. Breast Imaging Reporting and Data System (BI-RADS) category 4 and 5 mammograms and solid sonographic masses were considered suspicious for malignancy. Six of 165 men (4\%) had primary breast carcinoma, which were mammographically suspicious in all $6(100 \%)$. Five were invasive ductal carcinoma and one was ductal carcinoma in situ (DCIS). Of 164 mammograms, 20 (12\%) were suspicious. Six were cancer and 14 were benign. Clinical follow-up for 2 years or biopsy results were available for 138 of the 165 men (84\%). Twelve with benign mammographic findings had benign biopsies. All men with benign mammography not undergoing biopsy were cancer free. Sensitivity for cancer detection (mammography) was $100 \%$ and specificity was $90 \%$. Positive predictive value (mammography) was $32 \%$ (6 of 19) and the negative predictive value was $100 \%$. Sonography was performed in 68 of the 165 men (41\%). Three of three cancers (100\%) were solid sonographic masses. There were 9 of 68 false-positive examinations (13\%). Sensitivity and negative predictive value for cancer detection (ultrasound) was $100 \%$ and specificity was $74 \%$. The most common clinical indication for referral was mass/thickening (56\%). Mammography had excellent sensitivity and specificity for breast cancer detection and should be included as the initial imaging examination of men with clinical breast problems. The negative predictive value of $100 \%$ for mammography suggests that mammograms read as normal or negative need no further examination if the clinical findings are not suspicious. A normal ultrasound in these men confirms the negative predictive value of a normal mammogram.

Key Words: breast, male, mammography, ultrasound

A n estimated 1450 new cases of male breast cancer will be diagnosed in 2004 in the United States (1). Men present with breast cancer at a later stage than women $(2-4)$. However, the majority of symptomatic men will have benign abnormalities. Although most men with breast cancer present with a subareolar mass $(4,5)$, many men are referred for breast imaging because of other less specific clinical findings, such as tenderness or breast enlargement $(6,7)$. The algorithm for an accurate, cost-effective, and painless diagnosis of male breast cancer remains to be defined (5). Although the diagnostic accuracy of mammography in men appears very good for cancer detection (8), the literature is limited.

Address correspondence and reprint requests to: Stephanie K. Patterson, MD, Department of Radiology, University of Michigan Hospitals, TC2910B, 1500 E. Medical Center Dr., Ann Arbor, MI 48109-0326, USA, or e-mail: spatters@umich.edu.

(C)2006, Copyright the Authors

Journal compilation $@ 2006$, Blackwell Publishing, Inc., 1075-122X/06

The Breast Journal, Volume 12 Number 5, 2006 418-423
The purpose of our study was to determine the outcome of men presenting with clinical breast problems for breast imaging. We were especially interested in the role of mammography and ultrasound in the diagnosis of men with benign and malignant clinical breast problems.

\section{MATERIALS AND METHODS}

Institutional Review Board approval was obtained prior to commencement of the study. Individual patient informed consent for this retrospective study was not required. We retrospectively reviewed the clinical, radiographic, and pathologic records of 166 consecutive symptomatic men presenting to our Breast Imaging Section from June 1996 to August 2000. We assessed the reason for referral, mammographic findings, sonographic findings, histologic results, and clinical outcomes. Excluded was one man diagnosed with breast cancer at an outside institution where original mammograms are unavailable for review. In total, 165 breast imaging examinations 
were reviewed in 164 men. One hundred sixty-four mammograms were performed in 163 men, and 1 had an ultrasound only. One hundred forty-six had bilateral mammograms and 18 had unilateral mammograms. Patients ranged in age from 22 to 96 years, with a median age of 64 years. The clinical presentation of men described by the patient or the referring physician was recorded and categorized into five groups: 1) a lump or mass, 2) tenderness or pain, 3) “gynecomastia” or breast enlargement, 4) thickening, and 5) nipple discharge.

Mammograms performed at our institution were performed using standard film screen techniques on mammographic units (DMR General Electric Systems, Milwaukee, WI). Standard examination included craniocaudal, mediolateral oblique, lateral, and spot compression views with metallic markers over the palpable areas if a discrete lump was identified by breast examination performed by our clinical breast examiners.

Mammograms were read by radiologists who were Mammography Quality Standards Act (MQSA) certified. In 2000, the range of experience was from 2 to 22 years (mean 8.6 years). Breast Imaging Reporting and Data System (BI-RADS) (9) category assessments were recorded for each mammographic examination based on the original report. BI-RADS 1, 2, and 3 were negative, benign, and probably benign, respectively; BI-RADS 4 and 5 were suspicious and highly suggestive of malignancy, respectively.

Sonography was performed using high-resolution ultrasound equipment (model 700, GE Medical Systems, Milwaukee, WI; ATL HDI 5000, Philips, Bothell, WA) with 7.5-13 MHz linear transducers and was exclusively physician performed. Any discrete palpable lump or thickening was sonographically evaluated at the discretion of the radiologist. In patients who had both a mammogram and ultrasound, the interpretations were performed by the same radiologist at the same time. The sonographic findings were categorized into three groups: $(A)$ negative if no discrete abnormality was found, (B) solid mass, or (C) cystic mass. Although not available at the time of the study, groups A and C correspond to the current American College of Radiology Ultrasound BI-RADS categories 1-3 and group B to American College of Radiology Ultrasound BI-RADS (10) assessment 4 and 5.

The BI-RADS 4 and 5 mammographic assessments and any solid sonographic mass were considered suspicious for malignancy and recommended for biopsy. Mammography BI-RADS categories 1-3 were considered negative. Negative sonograms and simple cysts were considered negative sonographic findings. Sensitivities, specificities, positive predictive value (PPV), negative predictive value (NPV), and accuracy for mammography and sonography were performed.

Tissue diagnosis was obtained by open surgical biopsy or fine-needle aspiration (FNA) and microscopic evaluation was accepted as a definitive diagnosis. Tumor size, axillary lymph node status, hormone receptor status, and family history were recorded for men with carcinomas. If no tissue diagnosis was obtained, clinical or image followup was required for at least 2 years after the time of the original complaint to be considered benign. Patients with less than 2 years of documented follow-up were considered lost to follow-up. The data were entered into an Excel (Microsoft Corp., Seattle, WA) spreadsheet.

\section{RESULTS}

The most common presentation was a mass or thickening in 92 cases $(56 \%)$. Tenderness or pain was described in 50 cases $(30 \%)$ (Table 1). Fourteen men ( $8 \%$ ) presented with "gynecomastia" or breast enlargement (Fig. 1) and $7(4 \%)$ with nipple discharge. Two patients were referred to breast imaging to evaluate breast findings noted on chest computed tomography $(\mathrm{CT})$; one after a motor vehicle accident and the other after staging for lung cancer. There was complete follow-up, either clinical or pathologic, in 138 cases $(84 \%)$. Thirty-three men $(20 \%)$ underwent biopsy: 29 excisional biopsies and 4 FNAs. There were six primary breast carcinomas ( $4 \%)$ : one ductal carcinoma in situ (DCIS) and five invasive ductal carcinomas. The size of the cancers including the case of DCIS ranged between $1.6 \mathrm{~cm}$ and $3.5 \mathrm{~cm}$ (mean $2.4 \mathrm{~cm}$ ). All carcinomas presented as a palpable mass or thickening.

Of the 144 men categorized as mammography BIRADS 1, 2, or 3, 118 (72\%) had benign biopsies or benign clinical follow-up (Table 2 ) and $26(16 \%)$ were lost to

Table 1. Clinical Findings versus Outcomes in 165 Men Undergoing Breast Imaging

\begin{tabular}{llllll}
\hline & \multicolumn{5}{c}{ Outcomes } \\
\cline { 2 - 6 } & Malignant & $\begin{array}{c}\text { Benign } \\
\text { biopsy }\end{array}$ & $\begin{array}{c}\text { Benign } \\
\text { follow-up }\end{array}$ & $\begin{array}{c}\text { Lost to } \\
\text { follow-up }\end{array}$ & Total \\
\hline Mass/thickening & 6 & 20 & 52 & 14 & $92(56 \%)$ \\
Gynecomastia/breast & 0 & 1 & 10 & 3 & $14(8 \%)$ \\
$\quad$ enlargement & & 5 & 37 & 8 & $50(30 \%)$ \\
$\begin{array}{l}\text { Tenderness/pain } \\
\text { Nipple discharge }\end{array}$ & 0 & 1 & 4 & 2 & $7(4 \%)$ \\
$\begin{array}{l}\text { Other } \\
\text { Total }\end{array}$ & 0 & 0 & 2 & 0 & $2(1 \%)$ \\
& $6(4 \%)$ & $27(16 \%)$ & $105(64 \%)$ & $27(16 \%)$ & 165 \\
\hline
\end{tabular}



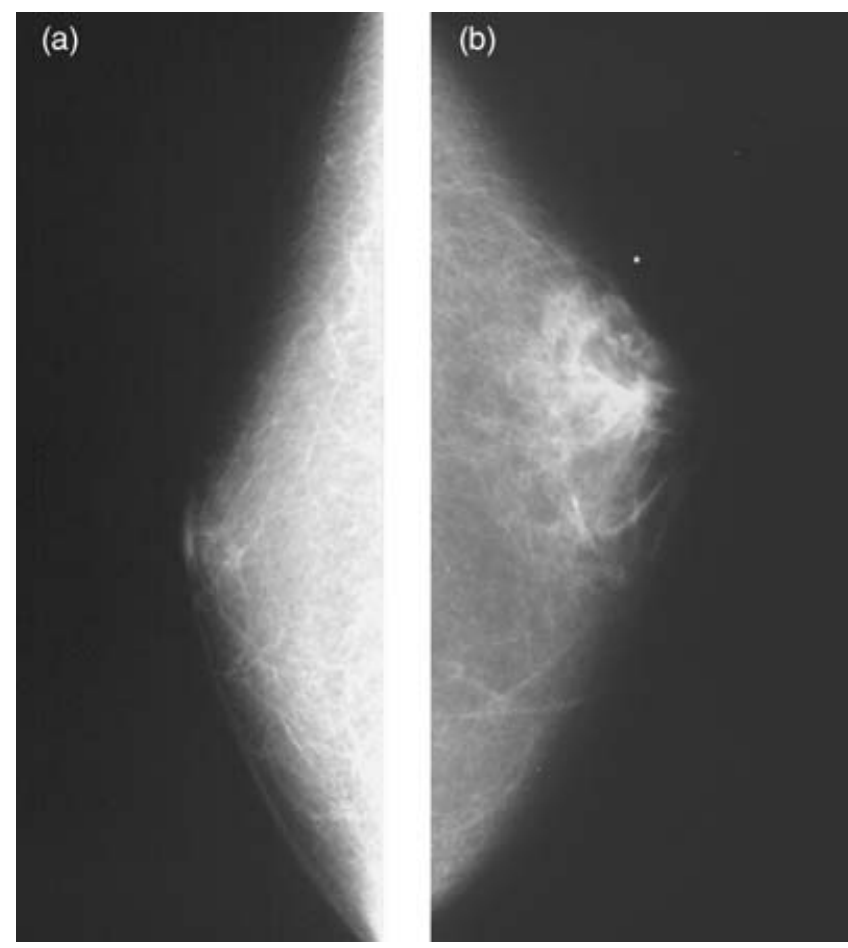

Figure 1. Examples of a normal or fatty male breast and gynecomastia. (a) A 57-year-old man with right nipple tenderness. Right craniocaudal view mammogram demonstrates fatty parenchyma without mass or other abnormality. Negative mammogram. (b) A 49year-old man presenting with left breast soreness and nodularity. Left craniocaudal mammogram demonstrates retroareolar fibroglandular tissue without a discrete mass consistent with gynecomastia, a benign finding. Biopsy confirmed gynecomastia.

follow-up. Six of the 20 men $(30 \%)$ categorized as mammography BI-RADS 4 or 5 had biopsies positive for malignancy (Fig. 2). One of the 20 (5\%) was lost to follow-up. No man categorized as mammography BIRADS 1, 2, or 3 had breast carcinoma. Pathologic results for the 17 men categorized mammography BI-RADS 1-3 who underwent biopsy demonstrated gynecomastia in 5 , lipoma in 5 , postoperative fluid collection in 1 ,

Table 2. Mammographic Assessment versus Clinical Outcome in 164 Men Undergoing Mammographic Evaluation

\begin{tabular}{lllccc}
\hline BI-RADS & Cancer & $\begin{array}{c}\text { Benign } \\
\text { biopsy }\end{array}$ & $\begin{array}{c}\text { Benign } \\
\text { follow-up }\end{array}$ & $\begin{array}{c}\text { Lost to } \\
\text { follow-up }\end{array}$ & Total \\
\hline 1,2 & 0 & 17 & 95 & 25 & $137(84 \%)$ \\
3 & 0 & 1 & 5 & 1 & $7(4 \%)$ \\
4,5 & 6 & 9 & 4 & 1 & $20(12 \%)$ \\
Total & $6(4 \%)$ & $27(16 \%)$ & $104(63 \%)$ & $27(16 \%)$ & 164 \\
\hline
\end{tabular}
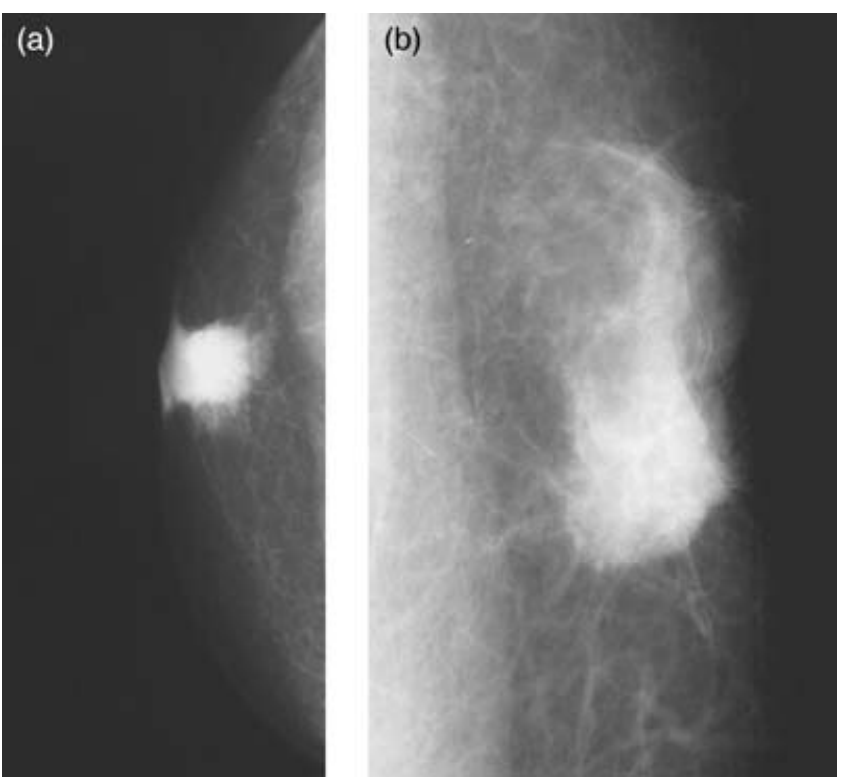

Figure 2. A 67-year-old man with a palpable, nontender, right breast mass positive for adenocarcinoma. (a) Right craniocaudal view mammogram demonstrates a round, spiculated, highly suspicious retroareolar mass without associated calcifications. (b) Close-up right lateral medial view mammogram of the retroareolar area demonstrating the highly suspicious mass.

negative FNAs in 4, dermatophytoses in 1, and fibrocystic change in 1 .

Sonography was performed in 68 cases $(41 \%)$. Fortyeight of these $(71 \%)$ were negative for a discrete mass, 19 $(28 \%)$ demonstrated a solid mass, and $1(1 \%)$ demonstrated a cystic mass. Sixteen of the 68 men who had sonographic evaluation were biopsied (24\%): 45 (66\%) had benign follow-up and $7(10 \%)$ were lost to follow-up (Table 3). Eleven of the 16 that were biopsied (68\%) had a solid mass on ultrasound (Fig. 3) and $4(25 \%)$ had negative ultrasounds. Three men with solid masses on ultrasound had carcinoma. No carcinoma was found in men with negative ultrasounds. There were nine false-positive

Table 3. Ultrasound Assessment versus Clinical Outcome in 68 Men Undergoing Sonographic Evaluation

\begin{tabular}{lllclc}
\hline Ultrasound & Cancer & $\begin{array}{c}\text { Benign } \\
\text { biopsy }\end{array}$ & $\begin{array}{c}\text { Benign } \\
\text { follow-up }\end{array}$ & $\begin{array}{c}\text { Lost to } \\
\text { follow-up }\end{array}$ & Total \\
\hline Negative & 0 & 4 & 39 & 5 & $48(71 \%)$ \\
Cystic mass & 0 & 1 & 0 & 0 & $1(1 \%)$ \\
Solid mass & 3 & 8 & 6 & 2 & $19(28 \%)$ \\
Total & $3(4 \%)$ & $13(19 \%)$ & $45(66 \%)$ & $7(10 \%)$ & 68 \\
& & & & & \\
\hline
\end{tabular}



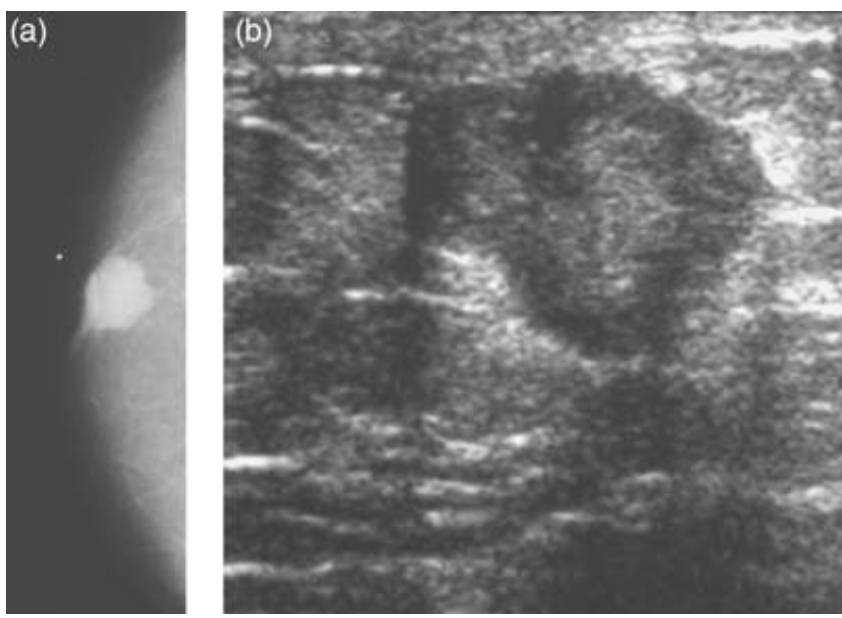

Figure 3. A 59-year-old man with a palpable left breast mass with nipple retraction; biopsy-proven invasive ductal carcinoma. (a) Left craniocaudal view mammogram demonstrating a retroareolar, lobulated, highly suspicious mass with spiculated margins without associated calcifications. (b) Ultrasound of the mass demonstrates a solid, lobulated mass.

sonographic examinations (13\%), including four gynecomastia (Fig. 4), two lipomas, one sinusoidal hemangioma, one postoperative fluid collection, and one benign FNA. Of the eight men with abnormal ultrasound findings who did not undergo biopsy ( 8 of $19 ; 42 \%$ ), six had no further breast complaints (including one patient with a lymph node confirmed by ultrasound) after 2 years and two were lost to follow-up (Table 3). The four men who had benign
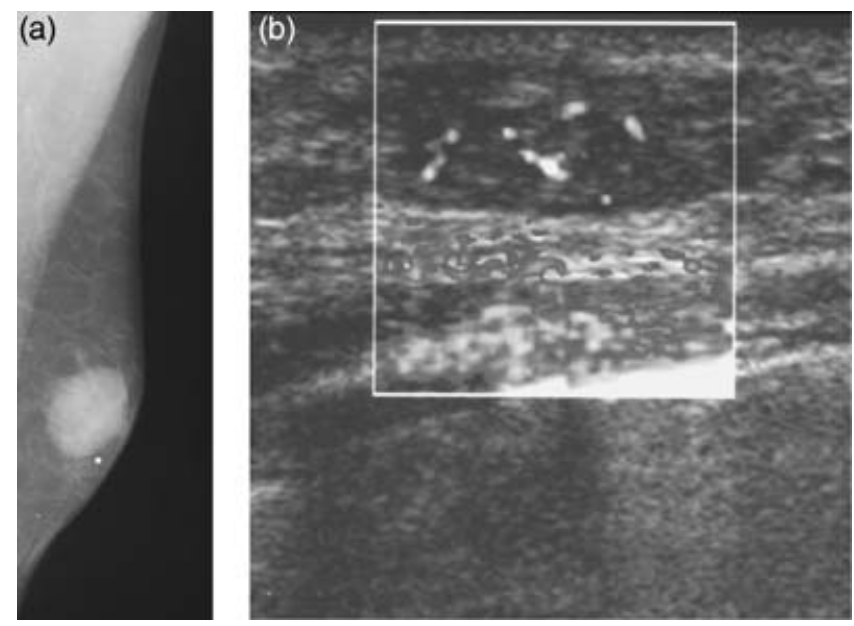

Figure 4. A 62-year-old man presenting with a palpable left breast mass; excisional biopsy proven gynecomastia. (a) Left mediolateral oblique view mammogram demonstrates a suspicious oblong retroareolar mass without associated calcifications. (b) Ultrasound with Doppler reveals a solid and vascular oblong retroareolar mass with indistinct margins.
Table 4. Mammography versus Ultrasound in 67 Men Undergoing Both Imaging Examinations

\begin{tabular}{lcccc}
\hline & \multicolumn{4}{c}{ Ultrasound findings } \\
\cline { 2 - 5 } Mammography & Normal & Solid mass & Cystic mass & Total \\
\cline { 2 - 5 } 1,2 & 42 & 7 & 1 & $50(75 \%)$ \\
3 & 3 & 1 & 0 & $4(6 \%)$ \\
4,5 & 2 & 11 & 0 & $13(19 \%)$ \\
Total & $47(70 \%)$ & $19(28 \%)$ & $1(1 \%)$ & 67 \\
\end{tabular}

biopsies and a negative ultrasound included three lipomas and one gynecomastia.

Of the 54 men categorized as mammography BI-RADS 1,2 , or 3 and evaluated with ultrasound, $45(83 \%)$ had negative sonograms, $8(15 \%)$ demonstrated a solid mass, and $1(2 \%)$ demonstrated a cystic mass (Table 4$)$. In 13 men categorized as mammography BI-RADS 4 or 5 , and evaluated with ultrasound, $2(15 \%)$ were sonographically negative and $11(85 \%)$ had sonographic masses (Table 4$)$. The single patient with sonographic evaluation only revealed a solid mass. This was the patient treated for an abscess that resolved. A single patient $(6 \%)$ with gynecomastia found on mammogram and categorized as BIRADS 4 demonstrated a solid sonographic mass, thus raising the level of suspicion.

\section{Tumor/Histology}

Five of the six men $(83 \%)$ with carcinoma had metastatic disease in sampled axillary lymph nodes. The single patient with negative sampled lymph nodes had papillary DCIS. When hormone receptor status was available, four $(67 \%)$ were estrogen receptor (ER) positive, three $(50 \%)$ were progesterone receptor $(\mathrm{PR})$ positive, one $(17 \%)$ was PR negative, one (17\%) was HER-2/neu positive, and three $(50 \%)$ were HER-2/neu negative. Two men had positive family histories for breast cancer and none were on medications or had exposures to known causes of gynecomastia. The median age of the men with breast carcinoma was 59 years (range $54-70$ years).

\section{Accuracy of Imaging}

The biopsy PPV for mammography was 32\% (6/19); the NPV was $100 \%(118 / 118)$. The sensitivity for cancer detection was $100 \%(6 / 6)$ and the specificity was $90 \%$ (118/131). The PPV for sonography was $17 \%(3 / 18)$; the NPV was $100 \%$ (43/43). The sensitivity for cancer detection was $100 \%$ (3/3) and the specificity was $74 \%$ (43/58). A palpable mass or thickening was the most sensitive 
clinical indicator, at $100 \%(6 / 6)$. The PPV of this finding was $8 \%(6 / 78)$.

\section{DISCUSSION}

The overwhelming majority of male breast problems are benign $(11,12)$ and include gynecomastia as one of the most common male breast complaints $(11,13)$. Although rare and accounting for less than $1 \%$ of all breast cancers (1), as in women, the incidence of breast carcinoma in men has increased (2). Breast cancer in men must be differentiated from benign clinical presentations. A later stage of disease at presentation is seen more often in men than in women $(3,14)$. However, men and women have equivalent prognoses when matched for age and stage of disease $(2,15)$. A proven appropriate algorithm for the evaluation of male breast problems has not been defined. FNA of palpable breast masses, together with physical examination, has proven accurate $(16,17)$. However, FNA can misdiagnose malignancies in the male breast (18). Evans et al. (8) found mammography alone has a sensitivity and specificity of at least $90 \%$ and a negative predictive value of $99 \%$. Given the small number of carcinomas found in men, a low-cost, accurate diagnostic test such as mammography could be an adjunctive tool to physical examination (8). Also, there has been very little in the literature on the role of ultrasound together with mammography in evaluating breast problems in men.

The most common mammographic finding in our series was gynecomastia, as in other reports $(6-8)$. No carcinoma was detected in men prospectively found to have gynecomastia on mammography or a normal mammogram, similar to a previous report (6). Gynecomastia has been described to be coexistent with breast cancer and obscuring it $(8,12,19)$, but this was not found in our population. All of the carcinomas in our series were retroareolar spiculated masses without calcifications on mammography. The mammographic assessment was predictive of clinical outcome. All malignancies were prospectively identified by mammography, confirming the high sensitivity of this modality.

Reports of ultrasound evaluation of male breast problems in the literature are limited, largely descriptive, and have limited numbers (11,20-23). Ultrasound complimented mammography in that the majority of sonograms were normal in men with mammography BI-RADS categories $1-3$, and the majority of sonograms demonstrated solid masses in men with mammography BI-RADS categories 4 or 5 . Ultrasound was found to successfully "downgrade" $15 \%$ (2 of 13) of false-positive mammographic cases (Table 4). No cancer was found in these cases if the ultrasound was negative. Ultrasound was very sensitive in our series, but lacked specificity.

In this series, men presented with a mass or thickening as the most common complaint, followed by breast tenderness or pain. This was similar to other reports $(6,11)$. A mass or thickening was the most sensitive indicator for breast carcinoma in our series, but lacked specificity, with a PPV of $8 \%$. Although some men with benign mammographic results were biopsied due to a high level of clinical suspicion, clinicians appear to use imaging to decrease the number of false-positive biopsies that would be generated by physical examination alone. A mammogram is recommended, given the high sensitivity of this modality, as the initial imaging examination if the clinical examination is not obvious. The NPV of $100 \%$ for mammography suggests that mammograms read as normal or negative need no further examination if the clinical findings are not suspicious. A normal ultrasound in these men confirmed the NPV of a normal mammogram. We did not find any carcinomas sonographically that were not detected on mammography. However, our series was too small to make a definitive statement regarding the role of ultrasound in male breast problems. Cases of carcinoma found by ultrasound that were obscured on mammography by gynecomastia have been described $(10,11)$. In women, it is the standard of care to perform ultrasound in the evaluation of a palpable abnormality without a discrete mammographic correlate (24), and it is likely prudent to do so in men.

The mean size of the carcinomas was $2.4 \mathrm{~cm}$, similar to other series $(2,11,19,20)$. The median age of 59 years of men with breast carcinoma in our series was lower than reported nationally $(2,25)$, and is likely due to our patient population and small sample size. All tumors were ductal in origin, as predicted (2). The single case of DCIS was of the papillary subtype, which has been described in more than $75 \%$ of DCIS cases in men (26). One of the most characteristic symptoms described for DCIS in men is serosanguinous nipple discharge (26). However, our one case of DCIS presented as a mass without nipple discharge. A markedly higher proportion of men with breast carcinoma have ER-positive tumors compared to women (2). When receptor status was known, $67 \%$ were ER positive compared to $90.6 \%$ nationally (2). All men with invasive tumors were lymph node positive, consistent with a higher rate of lymph node involvement at the time of diagnosis compared to women (2).

There are several limitations to our study. Even though our follow-up rate was reasonable, with $16 \%$ of men in 
this study lost to follow-up, our numbers remain small. Sonographic evaluation was performed after mammography and may have been influenced by the reader's knowledge of the mammogram. Also, we did not assess whether the routine craniocaudal and mediolateral oblique views are sufficient, and if the asymptomatic breast should also be imaged. Imaging of the asymptomatic breast may prove useful to assess for symmetry, but it has not been shown to be routine for screening contralateral asymptomatic breasts. We found no cases of bilateral breast cancer.

In conclusion, the imaging examination of men with clinical breast problems should include mammography, which appears to reduce the number of false-positive biopsies and showed excellent sensitivity, similar to an earlier report (8). Although the sensitivity of ultrasound was excellent as well, but with lower specificity, our numbers are too small to make a definitive recommendation to exclude ultrasound in the diagnostic evaluation of men with breast problems. As in women, ultrasound may be used to evaluate palpable abnormalities in men, particularly if there is gynecomastia, which could obscure an underlying malignancy.

\section{REFERENCES}

1. Jemal A, Tiwari RC, Murray T, et al. Cancer statistics, 2004. CA Cancer J Clin 2004;54:8-29.

2. Giordano SH, Cohen DS, Buzdar AU, Perkins G, Hortobagyi G. Breast carcinoma in men: a population-based study. Cancer 2004; 101:51-57.

3. Scott-Connor CEH, Jochimsen PR, Menck HR, Winchester DJ. An analysis of male and female breast cancer treatment and survival among demographically identical pairs of patients. Surgery 1999; 125:775-81.

4. Donegan WL, Redlich PN, Lang PJ, Gall MT. Carcinoma of the breast in males. Cancer 1998;83:498-509.

5. Meguerditchian A-N, Falardeau M, Martin G. Male breast carcinoma. Can J Surg 2002;45:296-302.

6. Cooper RA, Gunter BA, Ramamurthy L. Mammography in men. Radiology 1994;191:651-56.

7. Chantra PK, So GJ, Wollman JS, Bassett LW. Mammography of the male breast. AJR Am J Roentgenol 1995;164:853-58.

8. Evans GFF, Anthony T, Appelbaum AH, et al. The diagnostic accuracy of mammography in the evaluation of male breast disease. Am J Surg 2001;181:96-100.

9. American College of Radiology. Breast Imaging Reporting and Data System, 4th ed. Reston, VA: American College of Radiology, 2003.

10. American College of Radiology. Breast Imaging Reporting and Data System: Ultrasound. Reston, VA: American College of Radiology, 2003.

11. Gunhan-Bilgen I, Bozkaya H, Ustun EE, Memis A. Male breast disease: clinical, mammographic, and ultrasonographic features. Eur J Radiol 2002;43:246-55.

12. Appelbaum AH, Evans GFF, Levy KR, Amirkhan RH, Schumpert TD. Mammographic appearances of male breast disease. Radiographics 1999;19:559-68.

13. Braunstein GD. Gynecomastia. N Engl J Med 1993;328:49095 .

14. Ciatto S, Iossa A, Bonardi R, Pacini P. Male breast carcinoma. Review of a multicenter series of 150 cases. Tumori 1990;76:555-58.

15. Cutuli B, Lacroze M, Dilhuydy JM, et al. Male breast cancer: results of the treatments and prognostic factors in 397 cases. Eur J Cancer 1995;31A:1960-64.

16. Vetto J, Schmidt W, Pommier R, DiTomasso J, Eppich H, Wood W. Accurate and cost-effective evaluation of breast masses in males. Am J Surg 1998;175:383-87.

17. Joshi A, Kapila K, Verma K. Fine needle aspiration cytology in the management of male breast masses. Acta Cytol 1999;43:334-38.

18. Sneige N, Holder PD, Katz RL, et al. Fine-needle aspiration cytology of the male breast in a cancer center. Diagn Cytopathol 1993;9:691-97.

19. Dershaw DD, Borgen PI, Deutch BM, Liberman L. Mammographic findings in men with breast cancer. AJR Am J Roentgenol 1993;160:267-70.

20. Yang WT, Whitman GJ, Yuen EHY, Tse GMK, Stelling CB. Sonographic features of primary breast cancer in men. AJR 2001;176:413-16.

21. Rissanen TJ, Makarainen HP, Kallioinen MJ, Kiviniemi HO, Salmela PI. Radiography of the male breast in gynecomastia. Acta Radiol 1992;33:110-14.

22. Jackson VP, Gilmor RL. Male breast carcinoma and gynecomastia: comparison of mammography with sonography. Radiology 1983;149:533-36.

23. Stewart RAL, Howlett DC, Hearn FJ. Pictorial review: the imaging features of male breast disease. Clin Radiol 1997;52:739-44.

24. National Comprehensive Cancer Network. Clinical Practice Guidelines in Oncology. Breast Cancer Screening and Diagnosis. Jenkintown, PA: National Comprehensive Cancer Network, 2003.

25. Anderson WF, Althuis MD, Brinton LA, Devesa SS. Is male breast cancer similar or different than female breast cancer? Breast Cancer Res Treat 2004;83:77-86.

26. Hittmair AP, Lininger RA, Tavassoli FA. Ductal carcinoma in situ (DCIS) in the male breast: a morphologic study of 84 cases of pure DCIS and 30 cases of DCIS associated with invasive carcinomaa preliminary report. Cancer 1998;83:2139-49. 